\title{
Phase diagrams of $\mathrm{Si}_{1-\mathrm{x}} \mathrm{Ge}_{\mathrm{x}}$ solid solution: a theoretical approach
}

\author{
A.R. Jivani ${ }^{1}$ and A.R. Jani ${ }^{2}$ \\ ${ }^{1}$ V P and R P T P Science College, Vallabh Vidyanagar-388120, Gujarat, India \\ ${ }^{2}$ Department of Physics, Sardar Patel University, Vallabh Vidyanagar-388120, Gujarat, India \\ E-mail: jivani_a_r@yahoo.com \\ Phone: (office) +91-2692 - 230011; cell phone +91-9909012156; fax: +91-2692 235207
}

\begin{abstract}
In this work, we have used the pseudo-alloy atom model and higher-order perturbation theory based on pseudopotential approach to investigate phase diagram at different temperatures for $\mathrm{Si}_{1-x} \mathrm{Ge}_{x}$ solid solution system where $x$ is the arbitrary (atomic) concentration of the second constituting element. We have also investigated the phase diagram near the melting temperature as well as at low temperatures and compared with the available experimental results. Our calculated phase diagram near the melting point agrees well with the experimental data.
\end{abstract}

Keywords: solid solution, pseudopotential method, phase diagram.

Manuscript received 14.04.11; revised manuscript received 29.11.11; accepted for publication 26.01.12; published online 29.02.12.

\section{Introduction}

Group IV solid solutions such as $\mathrm{SiGe}, \mathrm{GeSn}$ etc. exhibit unique optoelectronic properties. The Group IV semiconductor alloys have wide (direct) bandgap materials, and hence they are used in fabrication of optoelectronic devices. These systems also improve the transport and optical properties as compared to its constituting atoms [1-5]. Therefore, the study of various physical properties of $\mathrm{Si}-\mathrm{Ge}$ system is very important both for experimentalists and theoreticians. The knowledge of phase diagrams for alloy systems is essential to understand behavior of alloys. In spite of this importance, only very little information about phase diagrams at and around the melting temperature are available for $\mathrm{Si}-\mathrm{Ge}$ system.

Previously, we have successfully employed our own proposed potential [6-10] to investigate a large number of physical properties of various semiconductor systems. We fruitfully reported the total energy, bulk modulus and heat of formation of $\mathrm{Si}-\mathrm{Ge}$ system [9] using our potential and hence this prompted us to make these calculations of phase diagrams at various temperatures with using our proposed pseudopotential [6-10].

\section{Simulation details}

\subsection{Total energy calculations}

For a covalent crystal with the diamond structure, if being based on the higher-order perturbation theory, the total energy per atom of the crystal [6-10] is given by
$E=E_{i}+E_{0}+E_{1}+E_{2}+E_{\mathrm{cov}}$.

In Eq. (1), $E_{i}$ is energy contribution due to ion-ion interactions, usually known as the Madelung energy, $E_{0}$ is the energy of the homogeneous electron gas which is a sum of the kinetic energy, exchange energy and correlation energy, $E_{1}$ is the first-order energy of the valence electron due to the pseudopotential, $E_{2}$ is the band structure energy, and $E_{\text {cov }}$ is the covalent-correction term. The covalent term is approximately a sum of third and fourth-order perturbation energies, which is essential while we study electronic, elastic and vibrational properties of semiconducting materials [6-10].

\subsection{Pseudo-alloy atom model}

In these investigations of phase diagrams for solid solutions, we have the used pseudo-alloy atom model (hereafter referred to as PAA) [11]. In this model, a hypothetical monatomic periodic lattice is replaced by the disordered alloy. The screened potential form factor $W_{s}^{P A A}(q)$ of an electron with a single PAA in the case of solid solution is given by

$$
\begin{aligned}
& W_{S}^{P A A}(q)=-\frac{12 \pi Z^{P A A} e^{2}}{\Omega q^{3} R_{c}^{P A A} \varepsilon(q)} \times \\
& \times\left[\frac{\sin \left(q R_{c}^{P A A}\right)}{2}-\frac{1}{q R_{c}^{P A A}}+\frac{\sin \left(q R_{c}^{P A A}\right)}{\left(q R_{c}^{P A A}\right)^{2}}\right],
\end{aligned}
$$

where $Z^{P A A}=Z^{\mathrm{Si}}=Z^{\mathrm{Ge}}=4$ is ion valency, $e$ is the electronic charge, $\Omega$ is the atomic volume, $q$ is the 
wave vector, and $\varepsilon(q)$ is the static Hartree dielectric function. The parameter of the potential at various concentrations $x$ is determined by

$$
R_{C}^{P A A}(x)=(1-x) R_{c}^{\mathrm{Si}}+x R_{C}^{\mathrm{Ge}} .
$$

The total energy $E^{P A A}(x)$ of the $\mathrm{Si}-\mathrm{Ge}$ solid solution is calculated using the higher-order perturbation theory [6-10] and with use of the above average screened potential (Eq. (2)) and potential parameter (as defined in Eq. (3)). In this work, we have used Sarkar et al. [12] local-field correction function to consider exchange and correlation effects on dielectric function.

\subsection{Heat of formation}

The heat of formation $\Delta E(x)$ for solid solutions is defined as the energy difference between the energy of solid solution $E^{P A A}(x)$ and the energy of phase mixture $E_{\text {mix }}(x)$ which is given by

$\Delta E(x)=E^{P A A}(x)-E_{\text {mix }}(x)$.

In the equation (4), the total energy of phase mixture of solid solutions for $\mathrm{Si}-\mathrm{Ge}$ is given by

$E_{\text {mix }}(x)=(1-x) E^{\mathrm{Si}}+x E^{\mathrm{Ge}}$.

In the equation (5), $E^{\mathrm{Si}}$ and $E^{\mathrm{Ge}}$ are the total energy per atom of Si and Ge calculated with the equation (1).

\subsection{Phase diagram at low temperatures}

In this work, we have investigated the phase diagram at low temperatures as well as at melting temperatures.

The Helmhöltz free energy $F_{S}(x, T)$ for the solid solution at the temperature $T$ (in $\mathrm{K}$ ) is given by [13]

$F_{S}(x, T)=E(x, T)+$

$+k T[x \ln (x)+(1-x) \ln (1-x)]$.

Then, the Helmhöltz free energy of formation $F_{S}^{\prime}(x, T)$ for the solid solution [13] is equal to difference between $F_{S}(x, T)$ and the energy of phase mixture $E_{\text {mix }}(x)$ which is given by

$$
\begin{aligned}
& F_{S}^{\prime}(x, T)=F_{S}(x, T)-E_{\text {mix }}(x)= \\
& =\Delta E(x)+k T[x \ln (x)+(1-x) \ln (1-x)] .
\end{aligned}
$$

Here, in deriving the equation (7), the vibrational contribution to the internal energy and the thermal entropy were assumed to be independent of the alloy composition and the configurational entropy was taken independent of the random distribution.

\subsection{Phase diagram near the melting temperature}

For solid solution, the Helmhöltz free energy $F_{1}(x, T)$ for the liquid phase at the temperature $T$ (in $\mathrm{K}$ ) is given by [13]

$$
\begin{aligned}
& F_{L}(x, T)=(1-x)\left(E^{\mathrm{Si}}+L^{\mathrm{Si}}\right)+ \\
& +x\left(E^{\mathrm{Ge}}+L^{\mathrm{Ge}}\right)-\Delta S_{m}(x) T+ \\
& +\Delta E_{1}(x)+k T[x \ln (x)+(1-x) \ln (1-x)] .
\end{aligned}
$$

Similar to a solid phase, we can write the Helmhöltz free energy of formation $F^{\prime}(x, T)$ for the liquid phase as given by

$$
\begin{aligned}
& F_{L}^{\prime}(x, T)=F_{L}(x, T)-E_{\text {mix }}(x, T)= \\
& =(1-x) L^{\mathrm{Si}}+x L^{\mathrm{Ge}}-\Delta S_{m}(x) T+ \\
& +\Delta E_{1}(x)+k T[x \ln (x)+(1-x) \ln (1-x)] .
\end{aligned}
$$

We have also taken into consideration all the assumptions as for the solid phase and we have also taken the heat of solution for the liquid phase $\Delta E_{1}(x)=0$.

In order to perform numerical calculations of the phase diagrams near the melting point, we also assumed that the entropy change in the course of melting $\Delta S_{m}(x)$ is

$\Delta S_{m}(x)=(1-x) \frac{L^{\mathrm{Si}}}{T_{m}^{\mathrm{Si}}}+x \frac{L^{\mathrm{Ge}}}{T_{m}^{\mathrm{Ge}}}$,

where $T_{m}$ and $L$ are the melting points and heat of fusion, respectively.

\section{Results and discussion}

\subsection{Phase diagram at low temperatures}

The presently investigated $F_{S}^{\prime}(x, T)$ for $\mathrm{Si}-\mathrm{Ge}$ system as a function of the concentration $(x)$ is shown for three typical temperatures in Fig. 1.

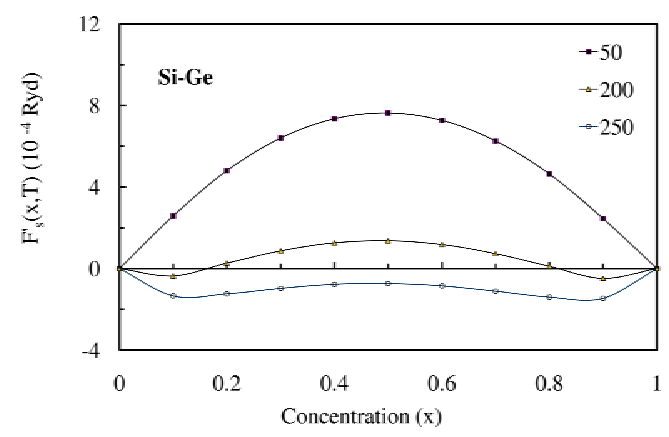

Fig. 1. The Helmhöltz free energy of formation $F_{S}^{\prime}(x, T)$ for $\mathrm{Si}-\mathrm{Ge}$ solid solution at 50,200 and $250 \mathrm{~K}$.

The Helmhöltz free energy of formation $F_{S}^{\prime}(x, T)$ for solid solutions is found to be positive as well as negative depending on temperature. Depending upon the 
sign, the physical interpretation may be formulated like this.

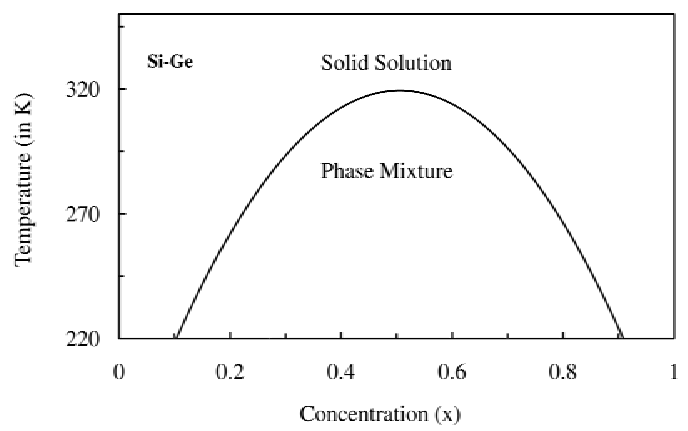

Fig. 2. The calculated phase diagram for $\mathrm{Si}-\mathrm{Ge}$ system at low temperatures.

The Helmhöltz free energy of formation $F_{S}^{\prime}(x, T)$ for $\mathrm{Si}-\mathrm{Ge}$ at $50 \mathrm{~K}$ is positive over the whole $x$ region as shown in Fig. 1. This suggests that the $\mathrm{Si}-\mathrm{Ge}$ system is insoluble at this temperature. The Helmhöltz free energy of formation $F_{S}^{\prime}(x, T)$ for $\mathrm{Si}-\mathrm{Ge}$ at $200 \mathrm{~K}$ is found to be positive as well as negative. This curve gives the values of concentration and temperature at which the phase will change. The negative value of the Helmhöltz free energy of formation $F_{S}^{\prime}(x, T)$ for $\mathrm{Si}-\mathrm{Ge}$ at $250 \mathrm{~K}$ over the whole $x$ range indicates that $\mathrm{Si}-\mathrm{Ge}$ system forms a stable solid solution.

Fig. 2 shows the calculated solubility limit of $\mathrm{Si}-\mathrm{Ge}$ system. The $\mathrm{Si}-\mathrm{Ge}$ system exists as phase mixture with 10 to $90 \%$ concentration of Ge. There exists a wide region of phase mixture at low temperatures.

\subsection{Phase diagrams near the melting temperature}

In Figs $3 \mathrm{a}$ to $3 \mathrm{c}$, the free energy curves $\left(F_{S}^{\prime}(x, T)\right.$ and $\left.F_{L}^{\prime}(x, T)\right)$ are shown for three typical temperatures, namely: 1200,1450 and $1700 \mathrm{~K}$ for $\mathrm{Si}-\mathrm{Ge}$ solid solution.

It is seen from Fig. 3a that two curves coincide at $x=1$ and $1200 \mathrm{~K}$. The free energy of solid phase is negative throughout the $x$-region. Thus, $\mathrm{Si}-\mathrm{Ge}$ system at $1200 \mathrm{~K}$ forms a solid phase. The free energy of a liquid phase is initially positive up to $x=0.54$ and then after it becomes negative.

The free energy curves $F_{S}^{\prime}(x, T)$ and $F_{L}^{\prime}(x, T)$ for solid and liquid phases crosses at $1450 \mathrm{~K}$ as shown in Fig. 3b. Hence, this graph gives information about phase boundaries of these two phases.

In Fig. 3c, two curves $F_{S}^{\prime}(x, T)$ and $F_{L}^{\prime}(x, T)$ coincide at $x=0$ and $1700 \mathrm{~K}$ temperature. The free energy of the solid phase as well as liquid phase is negative throughout the $x$-region. The value of $F_{S}^{\prime}(x, T)$ is more negative than $F_{L}^{\prime}(x, T)$ at a particular concentration, and this indicates that $\mathrm{Si}-\mathrm{Ge}$ system transforms to the liquid state.

The calculated phase diagram near the melting point is shown in Fig. 4 for $\mathrm{Si}-\mathrm{Ge}$ solid solution alongwith available experimental data [14]. From this figure, it is clear that the agreement of our calculation with the experimental data [14] is good.

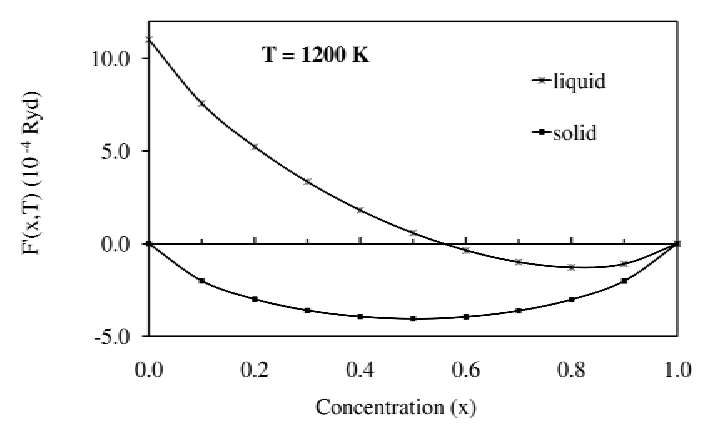

a

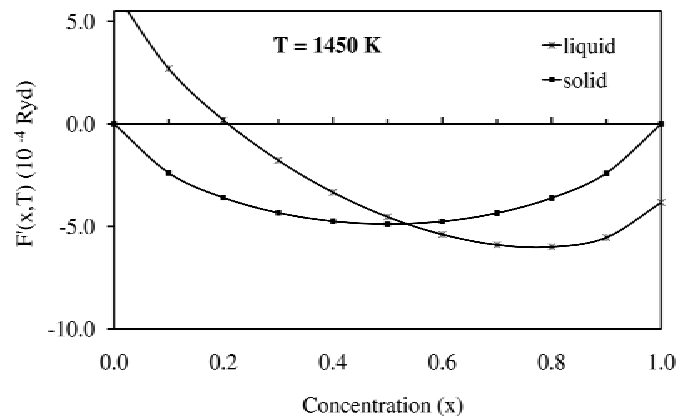

b

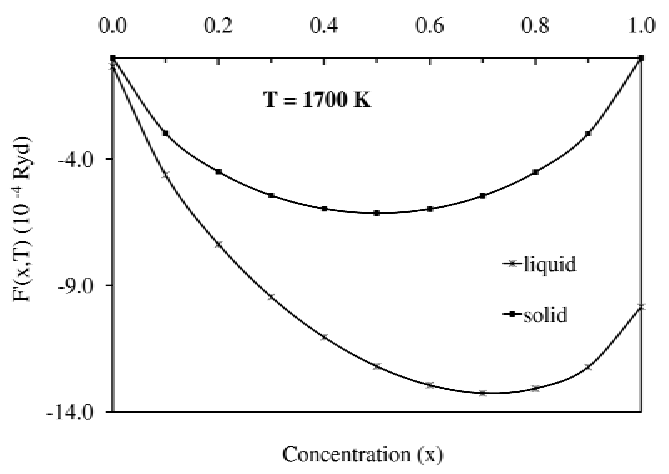

c

Fig. 3. The free energy curves for solid and liquid phases for $\mathrm{Si}-\mathrm{Ge}$ solid solution at 1200 (a), 1450 (b), and $1700 \mathrm{~K}$ (c). 


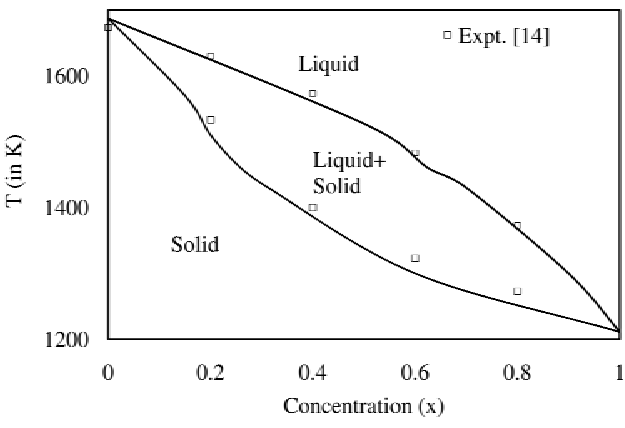

Fig. 4. The calculated phase diagrams near the melting points for $\mathrm{Si}-\mathrm{Ge}$ solid solution.

\section{Conclusions}

Thus, the phase diagram at various temperatures of $\mathrm{Si}-\mathrm{Ge}$ solid solution are studied employing the present formulation of the model potential [6-10] with the higher-order perturbation theory and the pseudo-alloy atom model [ $6-10$ ]. From the study of phase diagrams for the $\mathrm{Si}-\mathrm{Ge}$ system at low temperatures, we can conclude that there exists a wide region of phase mixture at low temperatures. It is also evident from this study that the calculated phase diagram near the melting point for $\mathrm{Si}-\mathrm{Ge}$ solid solution agrees well with the available experimental data [14]. Thus, our proposed potential [6-10] is efficient to study various properties of $\mathrm{Si}-\mathrm{Ge}$ solid solution, and hence this shows the strength of the potential.

\section{Acknowledgement}

A.R. Jivani thanks University Grants Commission, New Delhi, India, for financial support (Grant No. 47$625 / 08$ (WRO)) to carry out this research work.

\section{References}

1. A. Mesli, Vl. Kolkovsky, L. Dobaczewski, A. Nylandsted Larsen, N.V. Abrosimov, Defects and impurities in SiGe: The effect of alloying // Nucl. Instrum. and Methods in Phys. Research B, 253, p. 154-161 (2006).

2. V.J.B. Torres, J. Coutinho, P.R. Briddon, M. Barroso, Ab initio modeling of interstitial oxygen in crystalline SiGe alloys // J. NonCrystalline Solids, 352, p. 1279-1281 (2006).

3. Souraya Goumri-Said, Mohammed Benali Kanoun, Abdelkarim E. Merad, Ghouti Merad, Hafid Aourag, Empirical molecular dynamics study of structural, elastic and thermodynamic properties of zinc-blende-like SiGe compound // Mater. Sci. and Eng. B, 111, p. 207-213 (2004).
4. F. Ben Zid, A. Bhouri, H. Mejri, M. Said, N. Bouarissa, J.-L. Lazzari, F. Arnaud d'Avitaya, J. Derrien, Electronic structure and optical properties of $\mathrm{Si}_{1-\mathrm{x}} \mathrm{Ge}_{\mathrm{x}}$ alloys // Physica B, 322, p. 225-235 (2002).

5. N. Bouarissa, F. Annane, Electronic properties and elastic constants of the $\mathrm{Ge}_{1-\mathrm{x}} \mathrm{Sn}_{\mathrm{x}}$ alloys // Mater. Sci. and Eng. B, 95, p. 100-106 (2002).

6. A.R. Jivani, H.J. Trivedi, P.N. Gajjar and A.R. Jani, Some physical properties of $\mathrm{Si}_{1-\mathrm{x}} \mathrm{Ge}_{\mathrm{x}}$ solid solutions using pseudo-alloy atom model // SemiconductorPhysics, Quantum Electronics and Optoelectronics, 8, p. 14-17 (2006).

7. A.R. Jivani, H.J. Trivedi, P.N. Gajjar and A.R. Jani, Some physical properties of $\mathrm{GaX}(\mathrm{X}=\mathrm{P}$, As and $\mathrm{Sb}$ ) semiconductor compounds using higher-order perturbation theory // Physica B, 357, p. 305-311 (2005).

8. A.R. Jivani, H.J. Trivedi, P.N. Gajjar and A.R. Jani, Total energy, equation of state and bulk modulus of AlP, AlAs and AlSb semiconductors // Pramana - J. Phys. 64, p. 153-158 (2005).

9. A.R. Jivani, P.N. Gajjar and A.R. Jani, Application of pseudopotential to III-V semiconductor compounds // Indian Journal of Pure \& Applied Physics, 42, p. 833-836 (2004).

10. A.R. Jivani, P.N. Gajjar and A.R. Jani, Total energy, equation of states and bulk modulus of $\mathrm{Si}$ and Ge // Semiconductor Physics, Quantum Electronics and Optoelectronics, $\underline{5}$, p. 243-246 (2002).

11. T. Soma, The electronic theory of $\mathrm{Si}-\mathrm{Ge}$ solid solution // Phys. Status Solidi (b), 95, p. 427-431 (1979).

12. A. Sarkar, D. Sen, S. Haldar and D. Roy, Static local factor for dielectric screening function of electron gas at metallic and lower densities // Modern Phys. Lett. B, 12, p. 639-648 (1998).

13. http://www.doitpoms.ac.uk/tlplib/phase-diagrams

14. Sadao Adachi, Properties of Semiconductor Alloys: Group - IV, III-V and II-VI Semiconductors. Wiley, United Kingdom, 2005. 